\title{
C-Reactive Protein Monitoring Predicts Neutropenic Fever Following Autologous Hematopoietic Stem Cell Transplantation for Multiple Myeloma
}

Vidya Kollu ${ }^{1}$, Sarah L. Mott ${ }^{2}$, Rafiullah Khan ${ }^{3}$, Umar Farooq ${ }^{4}$, Yogesh Jethava ${ }^{3}$, Ince Dilek ${ }^{3}$ , Guido Tricot ${ }^{3}$

1. Internal Medicine, Medical College of Wisconsin, Milwaukee, USA 2. Biostatistics/Holden Comprehensive Cancer Center, University of Iowa, Iowa City, USA 3. Internal Medicine, University of Iowa, Iowa City, USA 4. Internal Medicine, University of Iowa Hospitals and Clinics, Iowa City, USA

$\square$ Corresponding author: Vidya Kollu, vkollu@mcw.edu

Disclosures can be found in Additional Information at the end of the article

\section{Abstract}

\section{Background}

Neutropenic fever (NF) is a known and common complication of autologous hematopoietic stem cell transplantation (ASCT). Early risk assessment may help direct treatment. We retrospectively analyzed the role of serial serum C-reactive protein (CRP) levels in predicting NF and assessed the clinical value of CRP within 14 days after transplantation.

\section{Methods}

One hundred twenty-one multiple myeloma (MM) patients received 170 first and/or second ASCT between January 2014 and March 2017. A Cox regression model was applied to assess the prognostic value of CRP as a time-dependent covariate at the onset of NF within 14 days posttransplant.

\section{Results}

Forty-seven of 170 patients developed NF. High CRP levels (4.0-43.2 mg/dL) were associated with a 5.45-fold increased risk of NF $(\mathrm{P}=0.02)$. Patients had a nearly three-fold increased risk of NF after the second transplant $(\mathrm{P}<0.01)$, but this was not associated with increased mortality. Those with NF had higher maximum values of CRP $(\mathrm{P}<0.01)$ which tended to occur at or after the onset of NF.

Received 06/09/2018 Review began 06/17/2018 Review ended 07/06/2018 Published 07/08/2018

\section{(c) Copyright 2018}

Kollu et al. This is an open access article distributed under the terms of the Creative Commons Attribution License CC-BY 3.0., which permits unrestricted use, distribution, and reproduction in any medium, provided the original author and source are credited.

\section{Conclusion}

CRP monitoring provides important information about the risk for NF immediately after first MM ASCT, and even more so after the second.

Categories: Infectious Disease, Oncology, Transplantation

Keywords: c-reactive protein, adult, hematopoietic stem cell transplantation, multiple myeloma, febrile neutropenia

\section{Introduction}

\section{How to cite this article}

Kollu V, Mott S L, Khan R, et al. (July 08, 2018) C-Reactive Protein Monitoring Predicts Neutropenic Fever Following Autologous Hematopoietic Stem Cell Transplantation for Multiple Myeloma. Cureus 10(7): e2945. DOI 10.7759/cureus. 2945 
Multiple myeloma (MM) is characterized by the proliferation of clonal bone marrow plasma cells and a monoclonal immunoglobulin or light chain overproduction with evidence of endorgan damage [1]. MM represents $1.8 \%$ of all new cancer cases in the US annually [2] and is the second most common hematologic malignancy, affecting about four in 100,000 [3]. Hematopoietic stem cell transplantation (HSCT) refers to the administration of hematopoietic progenitor cells from any source (e.g., bone marrow, peripheral blood, umbilical cord blood) or donor (e.g., allogeneic, autologous) to promptly reconstitute bone marrow function after highdose chemotherapy. Autologous HSCT (ASCT) uses hematopoietic progenitor cells derived from the patient. High-dose chemotherapy plus ASCT has been the standard treatment for newly diagnosed transplant-eligible MM adults up to 65 years of age [4]. In 2016, 8776 myeloma autologous transplants were reported to the Center for International Blood and Marrow Transplant Research. MM is currently the main indication for ASCT in Europe and the US [5, 6]. Tandem transplantation shows a higher overall survival (OS) and progression-free survival (PFS) when compared to patients who receive a single ASCT [7, 8].

After an ASCT, infection is the second most commonly reported cause of death, representing $15 \%$ of all fatalities, with disease relapse representing $69 \%$ of deaths [9]. Despite the many advances in supportive care, infections still continue to complicate the post-transplant course in many patients [10]; however, it is difficult to distinguish infection from other causes of fever, especially engraftment fever. The correct diagnosis of these major transplantation-related complications is essential for the early and proper administration of antibiotics or immunosuppressive treatment. The signs and symptoms of infection may be significantly muted in neutropenic patients or overlap with engraftment; thus, it is important to make use of objective evidence in the form of biomarkers to predict neutropenic fever (NF).

C-reactive protein (CRP) is an acute-phase protein produced by hepatocytes and is a reliable serum marker of infection, tissue destruction, necrosis and systemic inflammation [11-14]. While CRP testing is widely available and inexpensive, its use in day-to-day clinical decisionmaking is still limited and controversial.

Elevated CRP at diagnosis has a negative impact on myeloma prognosis [15]. Persistent elevation of CRP is an independent factor predicting fatal outcome in patients who remain febrile on the fifth day of antibiotic therapy during neutropenic febrile episodes post-HSCT [16]. CRP monitoring after blood and marrow transplant has identified patients at risk of severe transplant-related complications, mortality, and clinical outcomes [17, 18]. One recent study of the role of predictive biomarkers for cytokine release syndrome (CRS) after chimeric antigen receptor $\mathrm{T}$ cell therapy for acute lymphoblastic leukemia demonstrated that early CRP elevation was associated with grade 4-5 CRS ( $\mathrm{p}=0.02$ ), but was not useful in predicting CRS severity [19].

While many studies highlight the importance of CRP in detecting major transplant-related complications following HSCT, accurate guidelines for CRP monitoring remain unsettled. To our knowledge, no studies have reported the relevance of CRP in patients receiving tandem transplants for MM on prophylactic broad-spectrum antibiotics. We report our experience with CRP monitoring and attempt to delineate the significance of CRP monitoring in patients undergoing tandem ASCT for MM on prophylactic antibiotics.

\section{Materials And Methods}

\section{Study design}

This retrospective study included 170 consecutive ASCTs for MM, received by 121 patients between January 2014 and March 2017 at the University of Iowa. The study was approved by the University of Iowa Institutional Review Board and performed according to best clinical practices. Inclusion criteria included first or second transplant and availability of at least one 
CRP level post-transplant. The evaluation period was day zero to day 14 post-transplant. All patients received dexamethasone, cisplatin, adriamycin and etoposide (DPACE) induction therapy before collection of CD34 stem cells [20]. The majority started conditioning chemotherapy with VDT-melphalan $200 \mathrm{mg} / \mathrm{m}^{2}$ on day four before transplant [21]. Seventeen patients received melphalan $140 \mathrm{mg} / \mathrm{m}^{2}$ due to decreased kidney function (stage IV), one patient received VD-busulfan, and three received VD-melphalan $200 \mathrm{mg} / \mathrm{m}^{2}$.

Prophylactic and conditioning regimens were systematically applied according to our protocol. Antimicrobial prophylaxis with ciprofloxacin, fluconazole, and acyclovir was started four days prior to transplant, at admission. On day five post-transplant, patients were started on prophylactic meropenem intravenously [22]. Cytomegalovirus (CMV) polymerase chain reaction and aspergillus galactomannan antigen were checked weekly. Patients received granulocytecolony stimulating factor starting on day six. On day 12, darbepoetin alfa was given if hemoglobin was $<10 \mathrm{mg} / \mathrm{dL}$. During neutropenia, most patients remained hospitalized in an isolation unit with high-efficiency particulate air (HEPA) filtration.

Serum CRP levels were measured regularly during transplant, but only those obtained between days zero and 14 post-transplant were analyzed. Neutropenic fever was defined as having neutropenia and fever on the same day post-transplant. Neutropenia was defined as absolute neutrophil count (ANC) $<500 \mu \mathrm{L}$ or ANC level expected to fall below 500 over the next 48 hours. Prophylactic meropenem was used as a proxy for ANC expected to fall below 500 over the next 48 hours. Fever was defined as one temperature reading $>101^{\circ} \mathrm{F}$ or at least two temperature readings $>100.4^{\circ} \mathrm{F}$. Engraftment was defined as ANC $\geqslant 500$ for three consecutive days following HSCT. In the event of NF, blood cultures and chest X-ray were obtained and additional broadspectrum antibiotics initiated. Additional microbiological and radiological tests were obtained as clinically indicated. Blood cultures (one of each lumen of a central catheter plus one peripheral culture) were performed only with the first fever. For subsequent fevers, a blood culture was drawn only once every 24 hours via catheter. The need for peripheral cultures was individualized by patient per hospital protocol. Of the 170 transplants, 46 did not have a single ANC value from days zero through 14 due to non-availability of ANC values when a patient's total white blood cell count (WBC) was $<1,000 / \mu l$ attributable to lack of reliable reporting of a differential with such a low WBC count. Thus, NF was largely determined by additional intravenous antibiotic administration and elevated temperature. Of the remaining 124 transplants, the median number of ANC measurements was six. However, transplant patients had a median 13 CRP measurements. Temperature measurements were performed multiple times throughout the day, with median of 101 times per patient between days zero and 14 .

\section{Statistical analysis}

Continuous variables were summarized using the median and range (minimum and maximum). Categorical variables were summarized by count and percent. Logistic regression models were applied to assess differences between NF groups. An exchangeable correlation structure was used to model the possible dependency among multiple transplants within a single patient. To assess the prognostic effect of CRP on onset of NF within 14 days post-transplant, a Cox regression model was utilized. Time was calculated from transplant to onset of NF. CRP was categorized by quartiles and analyzed as a time-dependent covariate. A robust sandwich estimator of the standard error was applied to account for a clustering effect of multiple transplants within a single patient. Estimated effects are reported as hazard ratios (HRs) along with 95\% confidence intervals (95\% CIs). A mixed-effects regression model was used to assess for differences in maximum CRP within 14 days post-transplant between those with and without NF. All tests were two-sided and assessed for significance at the $5 \%$ level using SAS v9.4 (SAS Institute, Cary, NC). 


\section{Cureus}

\section{Results}

The study included 121 patients ( $94.2 \%$ were Caucasian, $5 \%$ were African American and $0.8 \%$ were Asian), 82 males (67.8\%) and 39 females (32.2\%). Median age was 61 years (range 38-76).

Patient and transplant characteristics are summarized in Table 1.

\begin{tabular}{|c|c|c|c|}
\hline \multirow[b]{2}{*}{ Covariate } & \multicolumn{2}{|c|}{ Neutropenic Fever } & \multirow[b]{2}{*}{ p-value } \\
\hline & No $N=123$ & Yes $\mathrm{N}=47$ & \\
\hline \multicolumn{4}{|l|}{ History of prior malignancy } \\
\hline No & $118(95.9)$ & 46 (97.9) & 0.41 \\
\hline Yes & $5(4.1)$ & $1(2.1)$ & \\
\hline \multicolumn{4}{|l|}{ CMV } \\
\hline Negative & $60(48.8)$ & $24(51.1)$ & 0.80 \\
\hline Positive & 63 (51.2) & $23(48.9)$ & \\
\hline \multicolumn{4}{|l|}{ Comorbidity index } \\
\hline High & $82(66.7)$ & $38(80.9)$ & 0.06 \\
\hline Intermediate-low & $41(33.3)$ & $9(19.1)$ & \\
\hline \multicolumn{4}{|l|}{ ASBMT risk } \\
\hline High & $31(32.3)$ & $11(26.2)$ & 0.37 \\
\hline Low & $65(67.7)$ & $31(73.8)$ & \\
\hline \multicolumn{4}{|l|}{ Preparative regimen } \\
\hline VD-busulfan & $1(0.8)$ & 0 & - \\
\hline VD-melphalan 200 & $2(1.6)$ & $1(2.1)$ & \\
\hline VDT-melphalan 140 & $12(9.8)$ & $5(10.6)$ & \\
\hline VDT-melphalan 200 & $108(87.8)$ & $41(87.2)$ & \\
\hline \multicolumn{4}{|l|}{ Transplant } \\
\hline First & 84 (68.3) & $19(40.4)$ & $<.01$ \\
\hline Second & $39(31.7)$ & $28(59.6)$ & \\
\hline \multicolumn{4}{|l|}{ Disease status at transplant } \\
\hline Complete response & $31(25.4)$ & $20(42.6)$ & 0.05 \\
\hline Not complete response & 91 (74.6) & 27 (57.4) & \\
\hline \multicolumn{4}{|l|}{ Performance status at transplant } \\
\hline KPS & 81 (66.4) & 31 (67.4) & 0.94 \\
\hline
\end{tabular}




\section{Cureus}

KPS-90

$41(33.6)$

$15(32.6)$

Best response post-transplant

Complete response

74 (73.3)

$33(82.5)$

Not complete response

$27(26.7)$

7 (17.5)

New malignancy post-transplant

$$
\begin{aligned}
& \text { No } \\
& \text { Yes }
\end{aligned}
$$

$121(98.4)$

$2(1.6)$

45 (95.7)

0.32

2 (4.3)

Age at transplant (years)

\section{Median \\ Range}

CD43 infused (million cells/kg)

Median

8.1

2.9-12.7

8.7

0.10

Range

ANC engraftment (days)

38-76
11

$7-28$
11

3.9-13.4

Range

TABLE 1: Patient characteristics and comparison of transplant characteristics by group.

CMV: Cytomegalovirus; ASBMT: American Society for Blood and Marrow Transplantation; KPS: Karnofsky Performance Scale; ANC: Absolute Neutrophil Count.

The median number of CD34 cells infused was 8.5 million $/ \mathrm{kg}$. The source of hematopoietic stem cells was peripheral blood in all cases. The median ANC engraftment day was day 11 (range 728). One patient with a high comorbidity index and low Karnofsky Performance Scale (KPS) died prior to engraftment during the second transplant due to infection and pulmonary toxicity. Of the 170 transplants, 47 developed NF (Table 1). NF occurred more often after second transplant (28 of 67, 42\%) compared to first transplant (19 of 103, 18\%). Though NF was more frequent after the second transplant, it was not associated with an increased mortality rate in the first 30 days. For patients who developed NF within 14 days post-transplant, engraftment occurred a median three days after the development of NF.

On multivariate analysis, high CRP levels (4.0-43.2 mg/dL) were associated with a 5.45-fold increased risk of NF $(\mathrm{P}=0.02)$ compared to undetectable levels $(<0.5 \mathrm{mg} / \mathrm{dL}$, Table 2$)$. 


\section{Cureus}

\begin{tabular}{|l|l|l|ll|l|}
\hline Variable & Comparison & Hazard Ratio & $95 \% \mathrm{Cl}$ & p-value \\
\hline CRP level & High $(4.0-43.2)$ vs undetectable $(<0.5)$ & 5.45 & 1.32 & 22.6 & 0.02 \\
& Low $(0.5-4.0)$ vs undetectable $(<0.5)$ & 2.38 & 0.70 & 8.13 & 0.17 \\
\multirow{2}{*}{ Transplant } & Second vs first & 2.98 & 1.73 & 5.13 & $<.01$ \\
\hline
\end{tabular}

TABLE 2: Comparison of C-reactive protein (CRP) levels.

Maximum CRP values were compared between those with and without NF. Patients developing NF post-transplant had higher maximum values of CRP compared to patients who did not develop NF $(\mathrm{P}<0.01$, Figure 1).

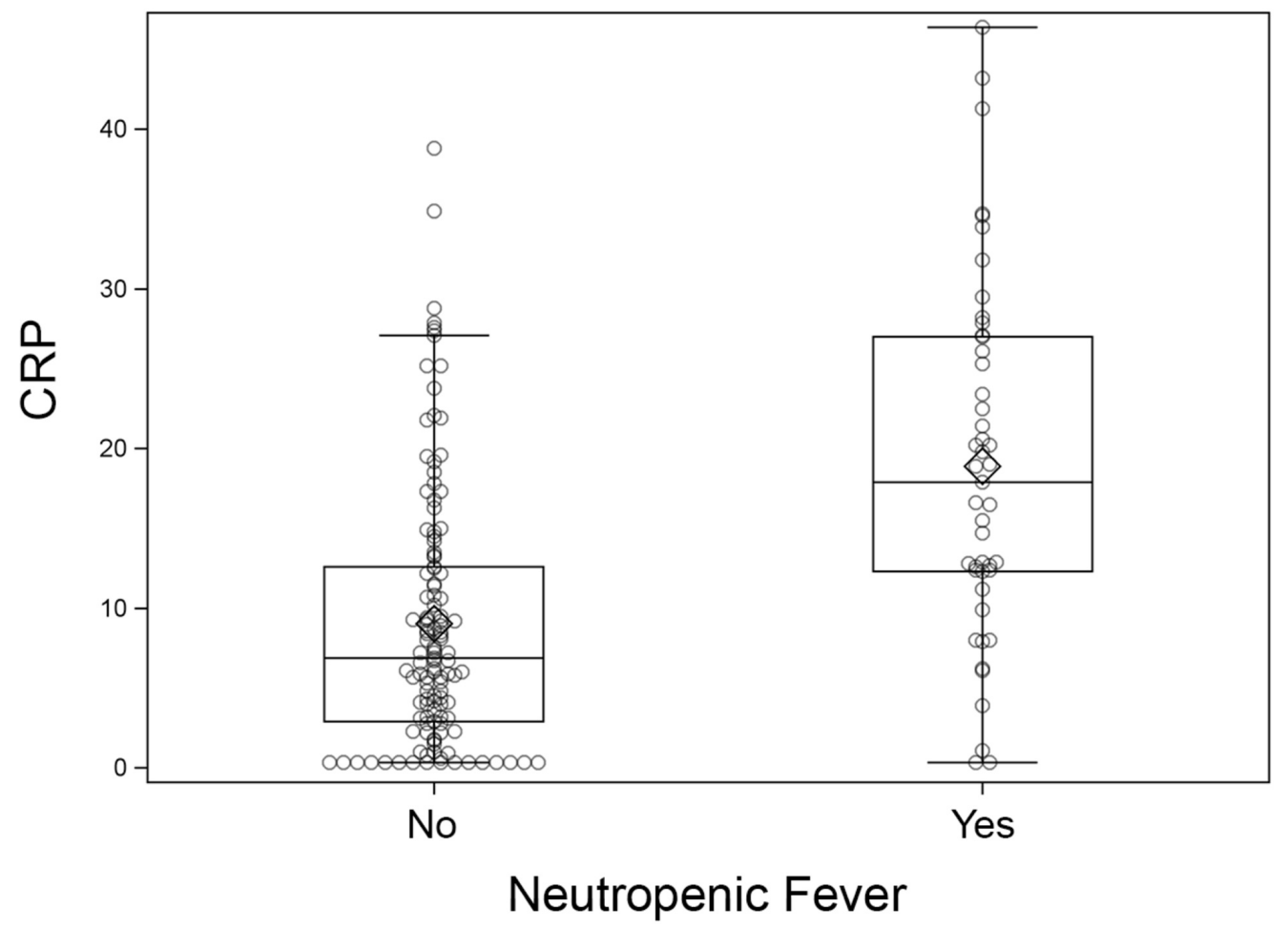

FIGURE 1: Maximum C-reactive protein (CRP) within 14 days of transplant.

Among those who developed NF, the maximum value of CRP was more frequently observed at the time or shortly after the onset of NF (35 of 47, 74\%).

\section{Discussion}

Our study demonstrated that in the presence of prophylactic broad-spectrum antibiotics, high CRP levels $(4.0-42.3 \mathrm{mg} / \mathrm{dL})$ were associated with a 5.45 -fold increased risk of NF $(\mathrm{P}=0.02)$ compared to undetectable levels $(<0.5 \mathrm{mg} / \mathrm{dL}$ ) following ASCT for MM. Patients receiving the second of tandem autologous transplants were at nearly three-fold increased risk of NF 
compared to the first transplant. Additionally, while high CRP levels were predictive of NF, levels frequently continued to increase after the onset of NF. NF most often occurred before engraftment; engraftment occurred a median three days after the onset of NF.

Serial CRP monitoring has been found to be valuable for the early diagnosis and monitoring of infections in neutropenic febrile children [23] and has reliably differentiated between bacterial infections and other causes of fever [24]. In one study, CRP was shown to be useful in monitoring the response to therapy for NF episodes, but did not predict NF or differentiate between the causes of fever [25]. Most reports to date have included relatively few patients, with various underlying diseases and therapies, including conventional and myeloablative chemotherapy with autologous or allogeneic transplant. The real frequency of bacteremia in our patients could not be assessed accurately because all patients were on empiric meropenem from day five.

Investigators have evaluated the impact of CRP levels on clinical outcomes in allogeneic HSCT. Sato et al. found that higher baseline CRP level was an independent risk factor for non-relapse mortality (NRM; HR: 6.21, P < 0.01), grade III-IV acute graft versus host disease (HR: 3.91, P = 0.03) and poor overall survival (HR: 3.27, $\mathrm{P}=0.0018$ ) [26]. Pavlu et al. reported that higher CRP levels independently predicted inferior survival and increased NRM in allogeneic HSCT for chronic myeloid leukemia [27]. Previous studies have examined inflammatory factors related to the development of high symptom burden during ASCT for MM. Wang et al. reported that serum CRP was significantly related to the most severe symptoms during the first 30 days after ASCT (P < 0.05) [28]. Fassas et al. demonstrated that a cut-off point of $100 \mathrm{mg} / \mathrm{L}(10 \mathrm{mg} / \mathrm{dL}) \mathrm{CRP}$ and $15 \mathrm{mg} / \mathrm{L} /$ day (CRP velocity) identified patients likely to suffer severe complications with 86\% and 75\% sensitivity, respectively [29]. Significantly higher mean CRP levels and CRP velocity of increase have also been observed among patients with severe complications [23]. Sato et al. reported that CRP levels immediately prior to first consolidation chemotherapy, but not before induction chemotherapy, had a significant predictive value for febrile neutropenia at a cut-off value of $0.19 \mathrm{mg} / \mathrm{dL}$ and documented infection at a cut-off value of $0.26 \mathrm{mg} / \mathrm{dL}$ [30].

Our study has certain limitations related to its retrospective nature. While high CRP levels were significantly associated with NF, we could not assess whether elevation in CRP could reliably distinguish between engraftment fever versus infection.

\section{Conclusions}

Early indication of NF helps identify those patients more likely to benefit from a combination of antibiotics while reducing unnecessary toxicity in those with low risk for NF. CRP monitoring provides important information about predicting NF during ASCT for MM, more so in tandem transplant. High CRP levels (4.0-43.2 mg/dL) were associated with a 5.45-fold increased risk of $\mathrm{NF}(\mathrm{P}=0.02)$. Patients had a nearly three-fold increased risk of NF after the second transplant $(\mathrm{P}<0.01)$, but this was not associated with increased mortality. Future prospective studies will help to elucidate the definitive role of CRP in identifying transplant patients at risk for NF and distinguishing engraftment fever from infections. Such results could be extrapolated to other transplant protocols for hematological malignancies.

\section{Additional Information}

\section{Disclosures}

Human subjects: Consent was obtained by all participants in this study. (University of Iowa) Hawk IRB issued approval 201704726. Animal subjects: All authors have confirmed that this study did not involve animal subjects or tissue. Conflicts of interest: In compliance with the ICMJE uniform disclosure form, all authors declare the following: Payment/services info: All 
authors have declared that no financial support was received from any organization for the submitted work. Financial relationships: All authors have declared that they have no financial relationships at present or within the previous three years with any organizations that might have an interest in the submitted work. Other relationships: All authors have declared that there are no other relationships or activities that could appear to have influenced the submitted work.

\section{Acknowledgements}

The authors would like to acknowledge Marilyn Rosenquist for assistance in submitting the IRB application, Jose Monestina and Lindsay Dozeman for their help with data collection, Heather Healy for help with literature searches and Kristina Greiner for assistance with manuscript editing.

\section{References}

1. Palumbo A, Anderson K: Multiple myeloma. N Engl J Med. 2011, 364:1046-1060. 10.1056/NEJMra1011442

2. SEER Cancer Statistics Review (CSR), 1975-2014 . (2018). Accessed: April 2017: https://seer.cancer.gov/archive/csr/1975_2014/.

3. Cruz RD, Tricot G, Zangari M, Zhan F: Progress in myeloma stem cells . Am J Blood Res. 2011, 1:135-145.

4. Child JA, Morgan GJ, Davies FE, et al.: High-dose chemotherapy with hematopoietic stem-cell rescue for multiple myeloma. N Engl J Med. 2003, 348:1875-1883. 10.1056/NEJMoa022340

5. Cavo M, Rajkumar SV, Palumbo A, et al.: International Myeloma Working Group consensus approach to the treatment of multiple myeloma patients who are candidates for autologous stem cell transplantation. Blood. 2011, 117:6063-6073. 10.1182/blood-2011-02-297325

6. Engelhardt M, Udi J, Kleber M, et al.: European Myeloma Network: the 3rd Trialist Forum Consensus Statement from the European experts meeting on multiple myeloma. Leuk Lymphoma. 2010, 51:2006-2011. 10.3109/10428194.2010.516378

7. Barlogie B, Jagannath S, Desikan KR, et al.: Total therapy with tandem transplants for newly diagnosed multiple myeloma. Blood. 1999, 93:55-65.

8. Barlogie B, Tricot GJ, van Rhee F, et al.: Long-term outcome results of the first tandem autotransplant trial for multiple myeloma. Br J Haematol. 2006, 135:158-164. 10.1111/j.13652141.2006.06271.x

9. Current uses and outcomes of hematopoietic cell transplantation (HCT): CIBMTR summary slides. (2017). Accessed: February 2018: https://www.cibmtr.org/ReferenceCenter/SlidesReports/SummarySlides/pages/index.aspx.

10. Fujii K, Aoyama M, Shinagawa K, et al.: Risk of neutropenic fever and early infectious complications after autologous peripheral blood stem cell transplantation for malignant diseases. Int J Hematol. 2002, 76:186-191. 10.1007/BF02982583

11. Colak A, Yilmaz C, Toprak B, Aktogu S: Procalcitonin and Crp as biomarkers in discrimination of community-acquired pneumonia and exacerbation of COPD. J Med Biochem. 2017, 36:122126. 10.1515/jomb-2017-0011

12. Gao LQ, Liu XH, Zhang DH, et al.: Early diagnosis of bacterial infection in patients with septicopyemia by laboratory analysis of PCT, CRP and IL-6. Exp Ther Med. 2017, 13:34793483. 10.3892/etm.2017.4417

13. Povoa P, Coelho L, Almeida E, Fernandes A, Mealha R, Moreira P, Sabino H: Early identification of intensive care unit-acquired infections with daily monitoring of C-reactive protein: a prospective observational study. Crit Care. 2006, 10:R63. 10.1186/cc4892

14. Morley JJ, Kushner I: Serum C-reactive protein levels in disease . Ann N Y Acad Sci. 1982, 389:406-418. 10.1111/j.1749-6632.1982.tb22153.x

15. Pineda-Roman M, Barlogie B, Tricot G, et al.: High-dose melphalan-based autotransplants for multiple myeloma: the Arkansas experience since 1989 in 3077 patients. Cancer. 2008, 112:1754-1764. 10.1002/cncr.23327

16. Ortega M, Rovira M, Almela M, de la Bellacasa JP, Carreras E, Mensa J: Measurement of Creactive protein in adults with febrile neutropenia after hematopoietic cell transplantation. 
Bone Marrow Transplant. 2004, 33:741-744. 10.1038/sj.bmt.1704409

17. Schots R, Kaufman L, Van Riet I, Lacor P, Trullemans F, De Waele M, Van Camp B: Monitoring of $\mathrm{C}$-reactive protein after allogeneic bone marrow transplantation identifies patients at risk of severe transplant-related complications and mortality. Bone Marrow Transplant. 1998, 22:79-85. 10.1038/sj.bmt.1701286

18. Sato M, Nakasone H, Wada H, et al.: Prediction of infectious events by the high-sensitivity Creactive protein level before autologous hematopoietic cell transplantation for lymphoma and multiple myeloma. Transpl Infect Dis. 2013, 15:169-171. 10.1111/tid.12102

19. Teachey DT, Lacey SF, Shaw PA, et al.: Identification of predictive biomarkers for cytokine release syndrome after chimeric antigen receptor T-cell therapy for acute lymphoblastic leukemia. Cancer Discov. 2016, 6:664-679. 10.1158/2159-8290.cd-16-0040

20. Ronchetti AM, Isnard F, Buffet M, et al.: Dexamethasone, cisplatin, doxorubicin, cyclophosphamide and etoposide (DPACE) is an effective salvage regimen for multiple myeloma refractory to novel agents. Leuk Lymphoma. 2013, 54:1117-1119.

10.3109/10428194.2012.733879

21. Nadiminti K, Singh Abbi KK, Mott SL, et al.: VTD-melphalan is well tolerated and results in very high rates of stringent $\mathrm{CR}$ and MRD-negative status in multiple myeloma. OncoTargets Ther. 2017, 10:217-226. 10.2147/ott.s112423

22. Kim JH, Goulston C, Zangari M, Tricot G, Boyer MW, Hanson KE: Impact of a change in antibacterial prophylaxis on bacteremia and hospitalization rates following outpatient autologous peripheral blood stem cell transplantation for multiple myeloma. Transpl Infect Dis. 2014, 16:421-429. 10.1111/tid.12225

23. Engel A, Mack E, Kern P, Kern WV: An analysis of interleukin-8, interleukin-6 and C-Reactive protein serum concentrations to predict fever, gram-negative bacteremia and complicated infection in neutropenic cancer patients. Infection. 1998, 26:213-221. 10.1007/bf02962366

24. Rintala E, Irjala K, Nikoskelainen J: Value of measurement of C-reactive protein in febrile patients with hematological malignancies. Eur J Clin Microbiol Infect Dis. 1992, 11:973-978. 10.1007/bf01967785

25. Yonemori K, Kanda Y, Togawa A, et al.: Clinical value of serial measurement of serum Creactive protein level in neutropenic patients. Leuk Lymphoma. 2001, 41:607-614. $10.3109 / 10428190109060351$

26. Sato M, Nakasone H, Oshima K, et al.: Prediction of transplant-related complications by Creactive protein levels before hematopoietic SCT. Bone Marrow Transplant. 2013, 48:698-702. 10.1038/bmt.2012.193

27. Pavlu J, Kew AK, Taylor-Roberts B, et al.: Optimizing patient selection for myeloablative allogeneic hematopoietic cell transplantation in chronic myeloid leukemia in chronic phase. Blood. 2010, 115:4018-4020. 10.1182/blood-2010-01-263624

28. Wang XS, Shi Q, Shah ND, et al.: Inflammatory markers and development of symptom burden in patients with multiple myeloma during autologous stem cell transplantation. Clin Cancer Res. 2014, 20:1366-1374. 10.1158/1078-0432.ccr-13-2442

29. Fassas AB, Miceli MH, Grazzlutti M, Dong L, Barlogie B, Anaissie E: Serial measurement of serum $\mathrm{C}$-reactive protein levels can identify patients at risk for severe complications following autologous stem cell transplantation. Leuk Lymphoma. 2005, 46:1159-1161. 10.1080/10428190500086121

30. Sato M, Kako S, Oshima K, et al.: Prediction of infectious events by high-sensitivity C-reactive protein level before undergoing chemotherapy for acute myeloid leukaemia. Scand J Infect Dis. 2010, 42:97-101. 10.3109/00365540903331019 